\title{
New Method to Estimation Electrical Stresses on 300kV OHTL/Cable Joints Due to Switching Operation Using ANN to Determine the Insulation Lifetime
}

\author{
Muhammad M.A.S. Mahmoud \\ Professor, IEEE Senior Member, Independent Researcher and Freelance Consultant, Cairo Egypt
}

\begin{abstract}
This article discuss the estimation of electrical stresses input that is required to determine lifetime degradation of the HV cables installed within overhead transmission lines (OHTL's). Abort from lightning effects, these electrical stresses are mainly generated due to repetitive switching operation of the transmission line that produces transient overvoltage (TOV) stress and high frequency stress. The transient overvoltage (TOV) due to switching-off the transmission line at the interface points between the cable and OHTL is estimated using artificial neural network (ANN). PSCAD software is used to build the transmission line model for 749 different case in order to build the required database for ANN training. Two different algorithm input to train ANN with one hidden layer and one output layer using different number of nodes are modeled in MATLAB. The results performance is continuously compared and evaluated till acceptable Regression is achieved to insure the error is less than $+/-1.5 \%$. The high frequency due to switching-on the transmission line is also recorded and analyzed. The results show that this new method is efficient, accurate and useful as there is no cable monitoring system is available for each HV cable installed within the transmission line.
\end{abstract}

Keywords. electrical insulation, lifetime, life span, HV cable, electrical stresses, ANN application,

\section{Introduction}

The main components in the HV Cables systems are cables, insulators joints, junction boxes, surge arrestors and termination kits. Any failure in the cable system components causes disturbance and/or interruption in the electrical power delivery process. A time is required to locate the faults, repair it and to restore the power back to clients. All this downtime can be translated into cost, loss of production and dissatisfaction to the clients [1-5].

Cable system lifetime Degradation and failures in most cases occurs due to operational reasons such as thermal overload, overvoltage stresses, high frequency stresses and/or mechanical damage. However, there are other factors that may play a role depending on the application. Manufacturing quality and installation conditions contribute in the failure rate [6].

Electrical current overloading and different types of short-circuit cases introduce heating energy in the cables proportional to the current square. This energy produces exponential temperature rise with time in the cable conductor material. In case the generated temperature rise rate is relatively high in such way that the heat dissipation from the cable is less than the heat rise in the insulation, this cause degradation of the insulation electrical properties. If the thermal overload cause is not eliminated in a very short time, coordinated with the insulation withstand, the cable insulation will damage. Also with increasing temperature, in additional to the generated mechanical stresses due to expansion process in the cable, the corrosive and oxidative effects on the insulation are increased. The speed of these degradation processes is not linear to the temperature and follows the so-called Arrhenius plot [7].

Sudden increase in voltage for the very short time in power system is known as the voltage surge or over voltage transients (TOV). This TOV can occur due to different external causes such as lightning, which 
impose fast-front overvoltage on the power system. Also, as the model circuits of cable systems and OHTL's consist of resistance, inductance and capacitance elements, therefore, switching-off operations either at normal condition or at short circuit conditions introduce overvoltage stresses on their insulation system. The wave form of the switching impulse has steep front and long damped oscillating tale.

In power system transmission line, during TOV electromagnetic waves propagate, reflect and refract through all the system and negatively affecting the power system insulation. The effect of the travelling waves is more harmful in case the OHTL transmission system have cables connected in between. The reason is that the characteristic impedance of OHTL lines is approximately 10 times, or even more, compared with the characteristic impedance of cables, and the lower the surge impedance the higher the time derivatives of the voltage generated in the OHTL/Cable joints. Such TOV and its wave propagation with high frequency cannot be avoided. This kind of voltage surges appear in the electrical power system due to switching surge is not very large in magnitude and hardly cross the twice of the normal voltage level. Generally, good insulation of the cable system and OHTL to the are sufficient to prevent damage due to this type of over voltages. However, repetition of OVT pulses, with its waves propagation and its high frequency, gradually degrade the insulations electrical properties and by time can damage the line [8 -12].

It is very important for the preventative maintenance team of the power system to estimate of the remaining lifetime for insulations operate under voltage and thermal stress, as this voltage can cause degradation in the insulation material electrical properties and shorten the insulation life span, which reduces the availability of the power network [13] [14].

One of the method to estimate the insulation material lifetime is to use the previous recorded data of operational conditions and failure and analyses it using different models such as Weibull and the CrowAMSAA to predict the lifetime of the insulation [15-17]. In this method, when utility engineers try to estimate the statistical time-to-failure for a given cable segment, the data should be interpreted correctly, and a sufficient number of data points is required to provide a reasonable level of confidence for the assessment of trends and predictions.

Another method is to install cable monitoring and management system to collect and analyze cables data and estimate their parameters and lifetime. This method is expensive and still under development. A combination of the monitoring method and historical data method is proposed but also under development [18].

The third method uses experiments that are carried out on the insulation material samples in laboratories under different conditions to produce graphs and equations that can be used to determine the lifetime of the that insulation material [19-20]. Different graphs and equations are produced to estimate the lifetime of the insulation material that are operated under repeated TOV condition. These methods need TOV amplitude, frequency and number of pulses as input [12] [21] [22]. In case of TOV generated by switching an OHTL has an interface point with cable, usually there is no metering system to capture the required input at the OHTL/cable joints. Some researches use the date collected by power analyzers to estimate the TOV at any point in the network and confirm the results using EMTP-RV software [23].

This article introduces new and effective method using ANN to determine the TOV and its frequency that electrically stress the insulation at OHTL/cable interface due to switching operation of $300 \mathrm{kV}$ transmission lines in order to provide the required input to estimate the electrical insulation remaining life span. In section II of this article, problem description and PSCAD modeling of the transmission line to calculate the TOV are provided. The generated data from PSCAD software and ANN modeling are introduced in Section III. The result and the conclusion are discussed in Section IV and V respectively. 


\section{Problem description and PSCAD modeling}

In general, underground cables are usually used in power system transmission and distribution within the capitals and big cities. Therefore, there is a need to connect the OHTL to cables before and after the city. Such design generates unavoidable OVT with nominal frequency at the interface point between OHTL and cables during switching-off the line, and generates higher frequency for the nominal voltage during switching on the line [24], which by time gradually degrade the insulation strength. This problem was observed by the electricity utility in one of the GCC capitals when they experienced frequently insulation failures at the OHTL/Cable joints of their $300 \mathrm{kV}$ network.

The steel tower structure design, the OHTL conductor data and the cable structure data for $300 \mathrm{kV}$ transmission system were obtained from the utility. It was found that the OHTL's and cables length that need to be included in the study changes from total line length from $5 \mathrm{Km}$ up to $125 \mathrm{~km}$, and the length of the cable section change from $0.5 \%$ to $5 \%$ of the total line and installed in the middle of the line.

In PSCAD model open circuit receiving end is assumed, as this presents the worst case for wave propagation that introduced the highest TOV as per Bewley Lattce diagram [25]. Figure 1 -3 illustrate the PSCAD model configuration for the transmission system under the study.

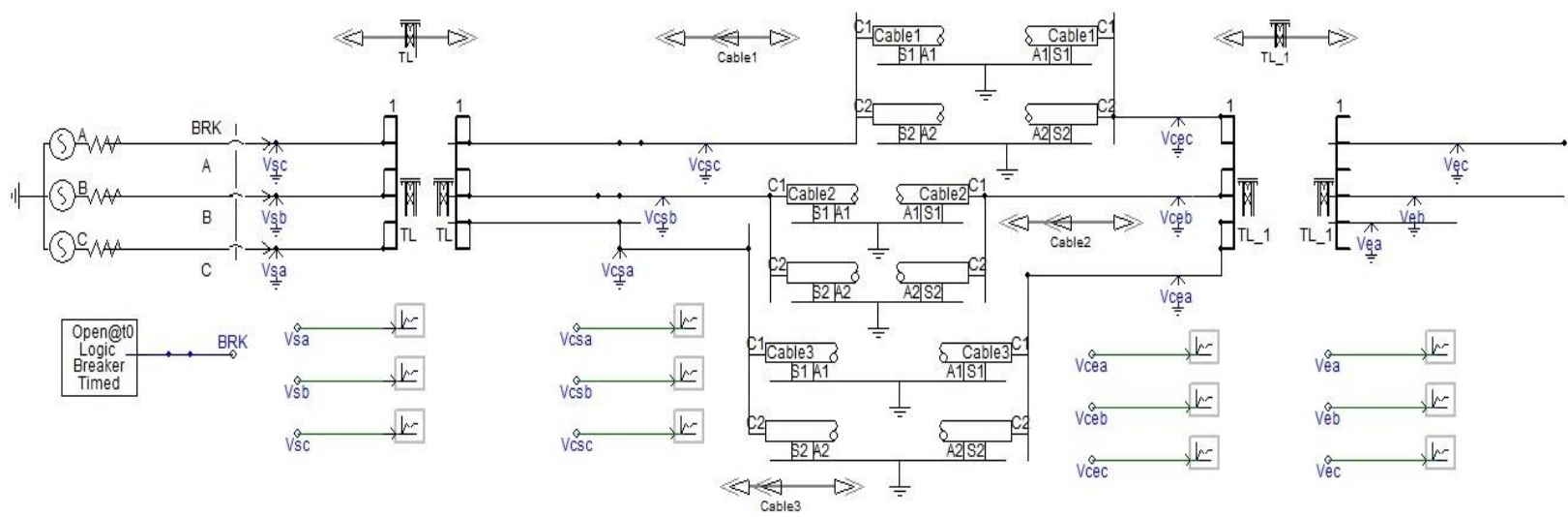

Figure 1: Transmission System Model

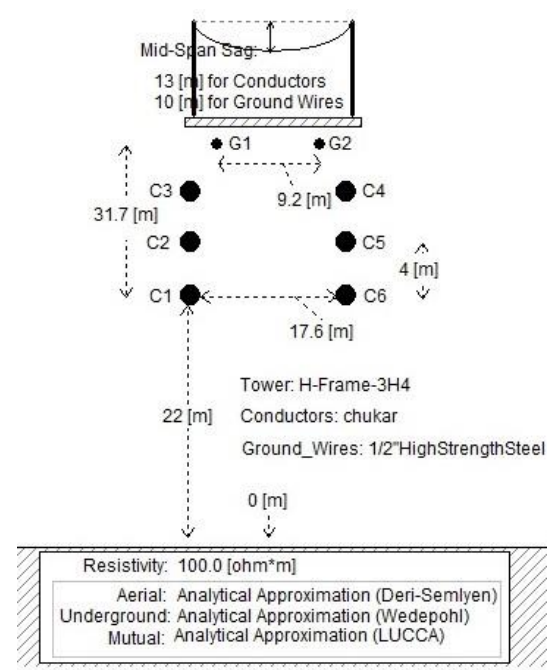

Figure 2: OHTL Model

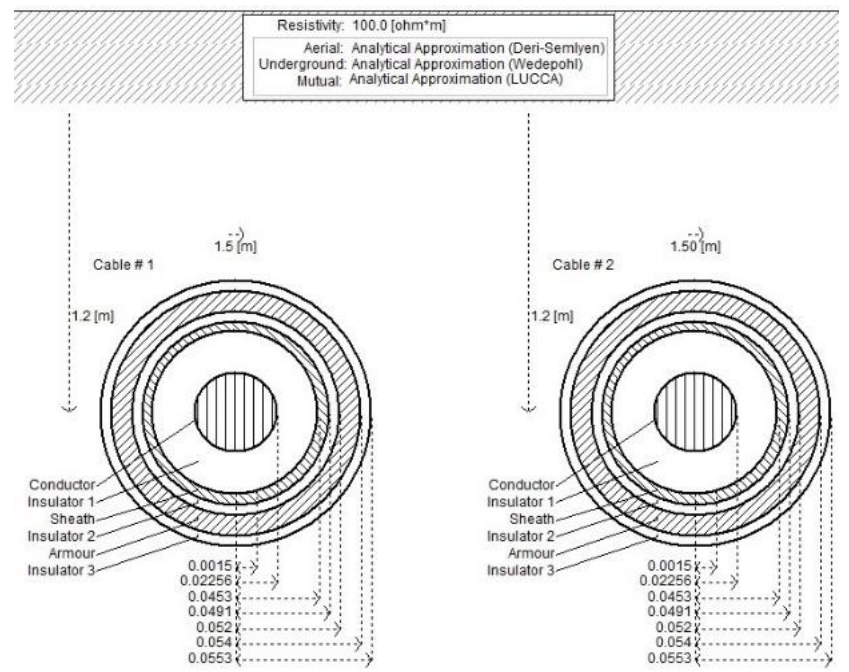

Figure 3: Cable Model 


\section{Database and ANN model Structure:}

\subsection{Database}

The PSCAD model, that is developed in section 2 for the power transmission system, is used to study 749 switching on/off cases represent different length combination of OHTL/cable configurations. In this comprehensive study TOV peak value at 3 different points are measured after simulating switching-off the transmission line; T1, T2 and T3, where T1 is the interface OHTL/Cable, T2 is the interface Cable/OHTL and T3 is the receiving end, seen from source side. Maximum frequency is recorded after switching-on the line.

\subsection{ANN model structure:}

The database obtained from PSCAD model is used to generate the input, target and testing data that required to train the proposed ANN model. Cable length, OHTL length and TOV at receiving end are selected as input to ANN because the transmission line configuration is always known and the TOV at T3 can be read from any power analyzer or any smart protection relay. The TOV at T1 and T2 are selected for Target. MATLAB software are used to build and train the ANN model.

For training, two different algorithms are used, BFGS Quai Newton and Bayesian Regularization. ANN for each algorithms consists of one hidden layer with (n) nodes and one output layer with 2 nodes, where $n$ starts from 5 and increased gradually in steps, each step equal one node, till obtain the acceptable Regression that insure the error is less than $+/-1.5 \%$. Eighty percent $(80 \%)$ of the data were used in the training process, and $20 \%$ of the data are used for testing. This method is applied with both training algorithms. Figure 4 and Figure 5 illustrate the ANN's that are used for both training algorithms. MATLAB default criteria to terminate each trial in both training algorithm is used for both algorithms.
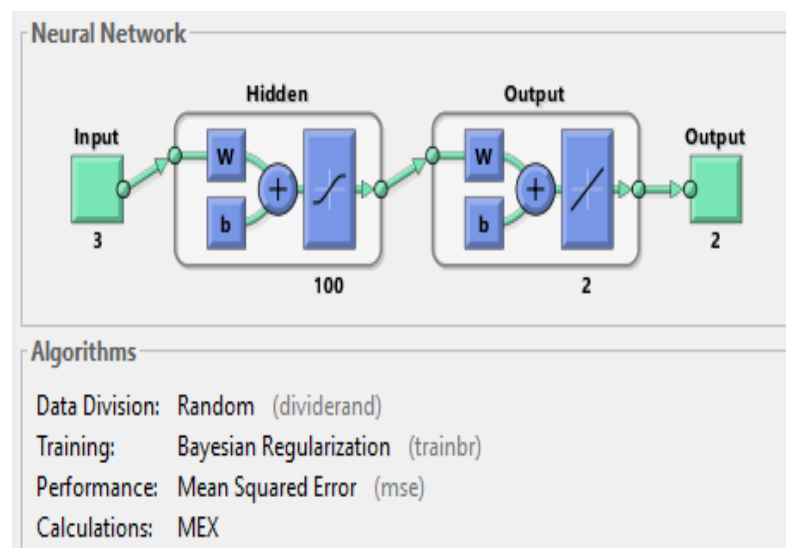

Fig.4: ANN with Bayesian Regularization algorithm

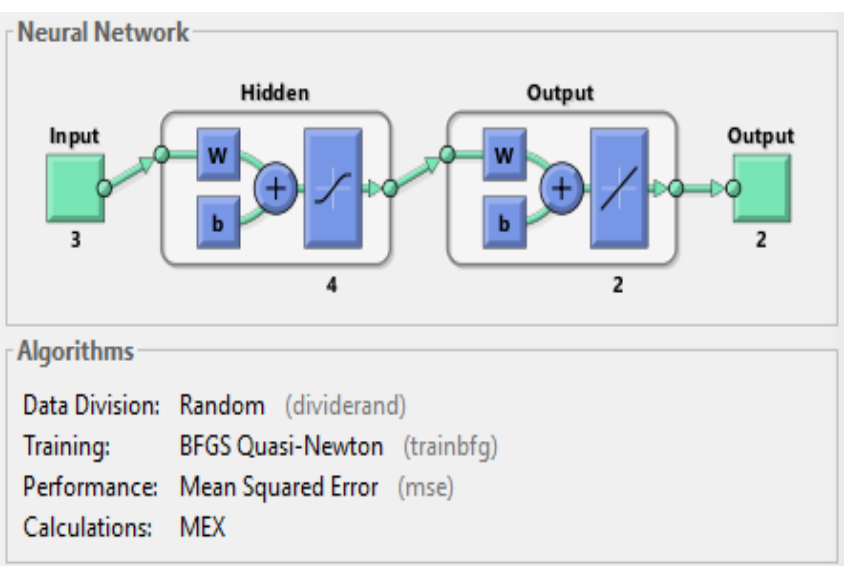

Fig.5: ANN with BFGS Quai Newton algorithm

\section{Results}

\subsection{Estimated TOV stresses:}

For the case of switching-off the line (TOV), the results of the trials for both algorithms are illustrated in Table 1. From the table, it is clear that the Bayesian Regularization Regression algorithm is faster and has achieved the satisfactory error by using 20 nodes with Total Data Regression of 0.99534 . While, BFGS Quai Newton algorithm has achieved Total-Data Regression of 0.99189 with same number of nodes. 
Table 1: Result of ANN trials from 5 nodes till 20 nods

\begin{tabular}{|c|c|c|c|c|c|c|c|c|}
\hline \multirow{2}{*}{$\begin{array}{c}\text { Number } \\
\text { of Nodes }\end{array}$} & \multicolumn{3}{|c|}{ BFGS Quai Newton Regression } & \multicolumn{4}{c|}{ Bayesian Regularization Regression } \\
\cline { 2 - 8 } & Training & Test & All & Performance & Training & Test & All & Performance \\
\hline 5 & 0.98368 & 0.98539 & 0.98448 & 17.8022 & 0.98853 & 0.98647 & 0.98814 & 13.621 \\
\hline 6 & 0.98605 & 0.98159 & 0.98578 & 16.3311 & 0.98858 & 0.98844 & 0.98853 & 13.1831 \\
\hline 7 & 0.98498 & 0.98727 & 0.98518 & 16.9953 & 0.99039 & 0.99019 & 0.99036 & 11.2625 \\
\hline 8 & 0.98534 & 0.98771 & 0.98526 & 16.9017 & 0.9911 & 0.98995 & 0.99086 & 10.515 \\
\hline 9 & 0.98648 & 0.98355 & 0.98576 & 16.33 & 0.992 & 0.98884 & 0.99135 & 9.9617 \\
\hline 10 & 0.98646 & 0.97581 & 0.98469 & 17.5575 & 0.99241 & 0.99064 & 0.99203 & 9.1874 \\
\hline 11 & 0.98653 & 0.988 & 0.987 & 14.9298 & 0.99271 & 0.99016 & 0.99222 & 8.9512 \\
\hline 12 & 0.98731 & 0.98559 & 0.98695 & 14.998 & 0.99301 & 0.99195 & 0.99276 & 8.3435 \\
\hline 13 & 0.98584 & 0.9781 & 0.98502 & 17.1786 & 0.99415 & 0.99198 & 0.99373 & 7.622 \\
\hline 14 & 0.98552 & 0.9858 & 0.98531 & 20.0864 & 0.99418 & 0.99088 & 0.99362 & 7.3496 \\
\hline 15 & 0.98762 & 0.98269 & 0.98732 & 17.1878 & 0.99414 & 0.99248 & 0.99376 & 7.1939 \\
\hline 16 & 0.98784 & 0.9873 & 0.98784 & 13.9695 & 0.99472 & 0.99155 & 0.99411 & 6.7876 \\
\hline 17 & 0.98759 & 0.98815 & 0.98742 & 14.652 & 0.99494 & 0.9931 & 0.99463 & 6.1878 \\
\hline 18 & 0.99023 & 0.98307 & 0.98906 & 12.5649 & 0.99534 & 0.99221 & 0.99476 & 6.0461 \\
\hline 19 & 0.9897 & 0.99137 & 0.98911 & 12.5145 & 0.99505 & 0.99374 & 0.99477 & 6.0317 \\
\hline 20 & 0.99193 & 0.99199 & 0.99189 & 8.7662 & 0.99566 & 0.99386 & 0.99534 & 5.3956 \\
\hline
\end{tabular}

Figure 6 illustrates the percentage error in the TOV at the interface points T1 and T2 obtained for the final ANN model using 20 nodes. The values in this Figure confirm that the percentage error is within the desired value $+/-1.5 \%$.

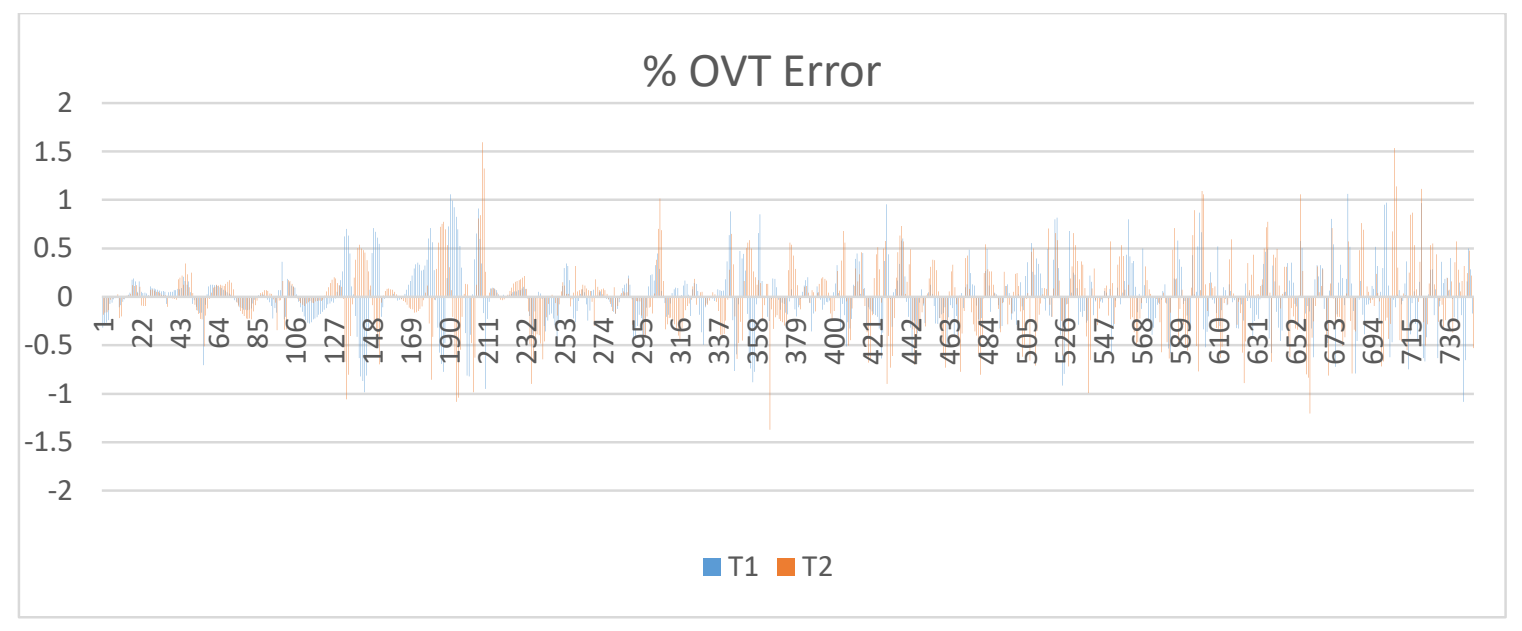

Fig 6: OVT error

The target values and the ANN output for T1 and T2 are plotted and illustrated on Figure 7 and 8. From these Figures, fitting of the ANN output with Target values can be noticed clearly. Also, as the data is listed starting from shortest cable ( $0.5 \%$ of OHTL) to the longest cable (5\% of OHTL), and this was repeated for OHTL lengths from 5 to $125 \mathrm{~km}$ in distance interval of $10 \mathrm{~km}$, the results show that TOV is higher when the cable is short. And, when the cable length is long as the OHTL length increases, the maximum TOV values at T1 and T2 decrease. But when the cable length is short, as the OHTL length increases, the maximum OVT values at $\mathrm{T} 1$ and $\mathrm{T} 2$ remain almost constant near to the value of $718 \mathrm{kV}$ and $730 \mathrm{kV}$ respectively. This means that cables with short length may have less lifetime due to switching operation. 


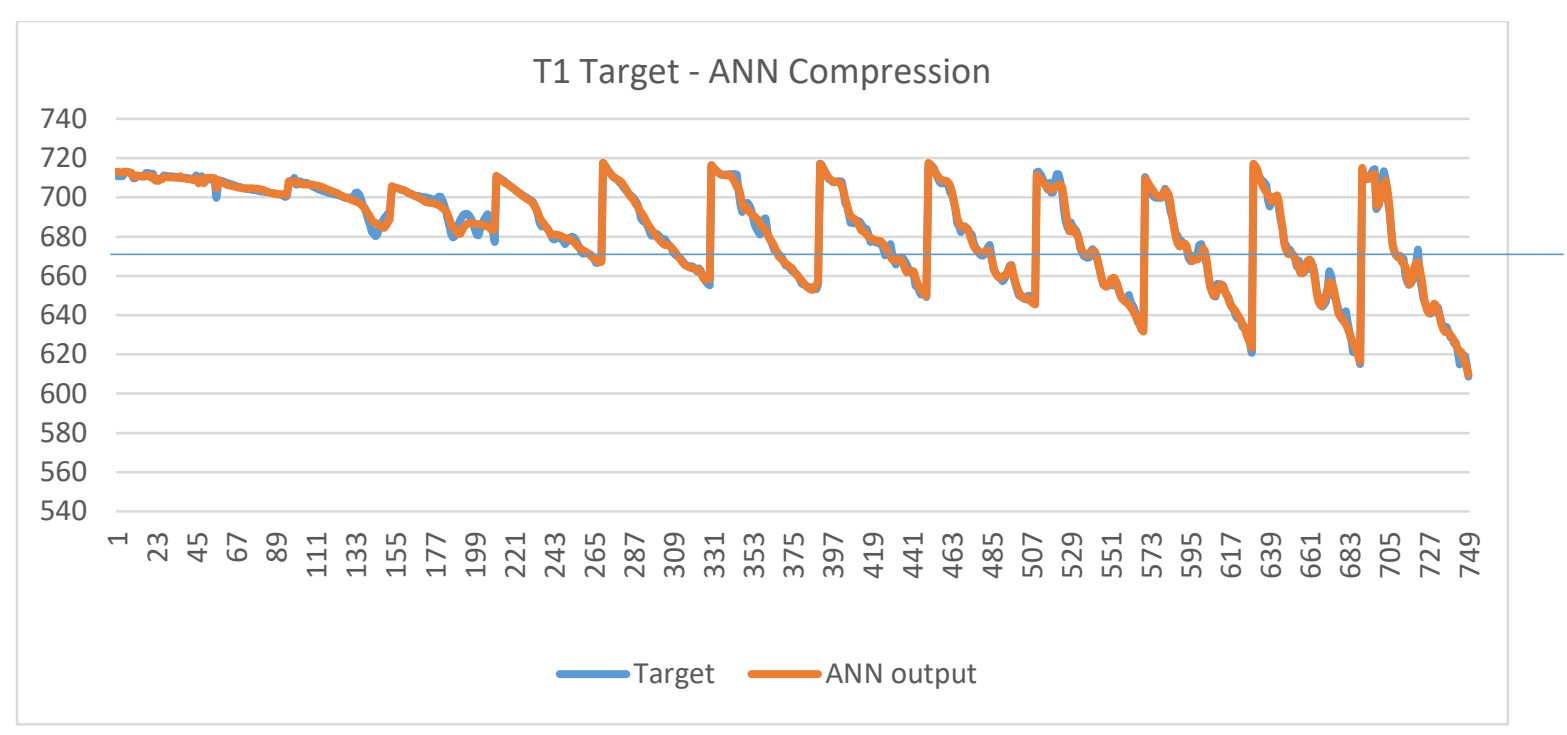

Fig. 7: Target - ANN at T1

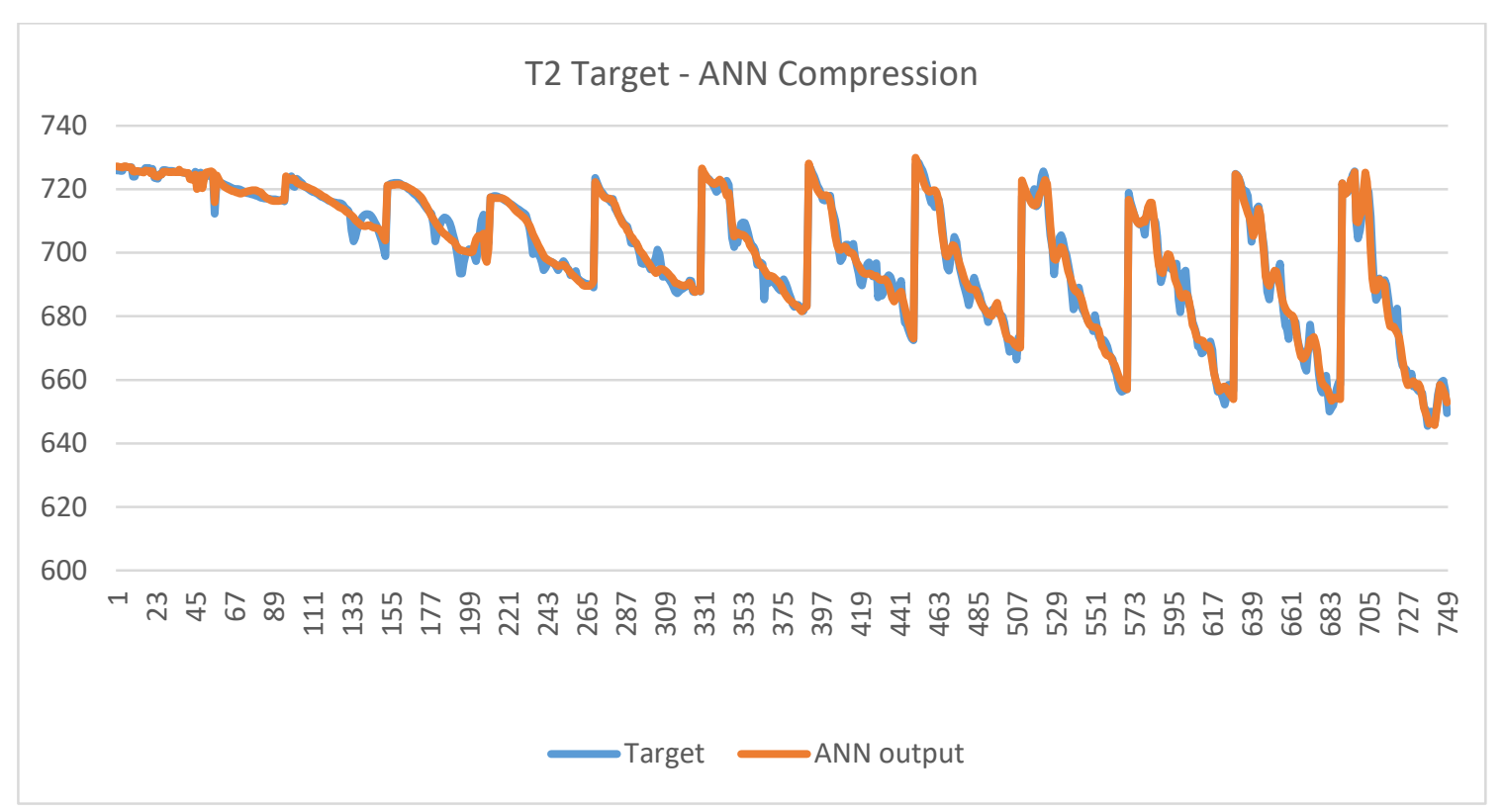

Fig. 8: Target $-\mathrm{ANN}$ at T2

The above ANN results shows acceptable accuracy that allows to use ANN to estimate the TOV stresses on the cable/OHTL joints based on the voltage measurement at receiving end. This method can be used with the number of the transmission line switching-off operations to determine the lifetime of the cable joints.

\subsection{Maximum Frequency:}

The frequency is recorded for 749 different cases after switch-on the line. It was noticed that, in general, for all cases the frequency reduces by increasing the cable length. This is because of the damping effect of the cable resistance, also the transient waves take more time to propagate between the two cable ends. The maximum frequency was recorded with a value of $193 \mathrm{~Hz}$, and it last for approximately for 100ms. (Figure 9). This frequency occurs with approximately nominal voltage. 


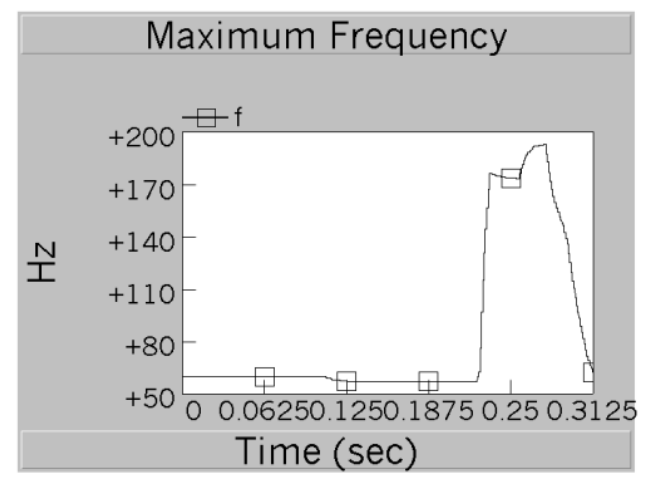

Fig. 9: Maximum frequency

A good approximation of the maximum frequency range represented by " $\Pi$ " line model is given by the following equation [26]:

Where,

$$
\begin{aligned}
f \max & =N \cdot \frac{v}{8 l} \\
v & =\frac{1}{\sqrt{L C}}
\end{aligned}
$$

- $N=$ Number of pi sections

$\bullet v=$ Propagation speed in $\mathrm{km} / \mathrm{s}$

$L$ in $\mathrm{H} / \mathrm{km}$,

$C$ in $\mathrm{F} / \mathrm{km}$

$\bullet l=$ Line length in $\mathrm{km}$

The above equation (1) is in line with the results obtained from PSCAD study for the relation between the frequency and the cable lengths.

As this maximum frequency value causes the maximum possible frequency stress on the cable insulation, so it can be used with the number of switching-on the line, to estimate the frequency stresses on the insulation and hence to calculate the lifespan of the cable.

\section{Conclusion}

This article discussed the electrical stresses input that required to determine the electrical insulation degradation of the HV cables installed within OHTL's due to stresses generated by repetitive switching on and off operations. As in typical design for transmission lines, there is no cable monitoring system is installed for each HV cable, and all measurements are usually taken at the substations, therefore, therefore there is a need to find am efficient method to estimate the TOV and high frequency that are generated on the OHTL/cable interfaces. In this article ANN is used to estimate the TOV stresses using one hidden layer and one output layer using 20 nodes. PSCAD software is used to build the required database (749 case) for ANN training, and to record the high frequency that is generated due to switching-on transmission lines. The proposed method uses available information that can be recorded and retrieved from any smart protection relay at the substations. The results and its analysis concludes that the proposed method is accurate, efficient and very useful to be used to estimate the required input to determine the insulation life time, and hence plan for effective maintenance plan for the transmission lines. 


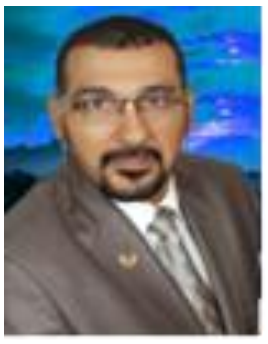

Muhammad M.A.S. Mahmoud, Egyptian, Received the B.S. degree in Engineering from Cairo University and the M.Sc. degree from Kuwait University. First Ph.D. degree from Transilvania University of Brasov, Romania in IT and Computer. Second PhD Degree in Electrical Power system and Machine, Cairo Univ. Egypt. He received a position of "Professor" on 2018. His current research interests in Fuzzy and Artificial Neural Network Techniques application include power delivery, protection reliability, control, safety, building automation and energy management. Prof. Dr. Muhammad is of IEEE Member in 1999, IEEE Senior Member (SM) since 2001 and TFS -IEEE Reviewer 2016.

References

[1] Muhammad M.A.S. Mahmoud, "Comparison of Different Clustering Validity Methods in the Evaluation of Results for Electrical Fault Location in Industrial MV Network Using Fuzzy Clustering Technique", 2020 6th IEEE International Energy Conference (ENERGYCon). DOI: 10.1109/energycon48941.2020.9236455

[2] Muhammad M.A.S. Mahmoud, "Review of Fuzzy and ANN Fault Location Methods for Distribution Power System in Oil and Gas Sectors", Elsevier IFAC-PapersOnLine 2018. https://doi.org/10.1016/j.ifacol.2018.11.298

[3] Muhammad M.A.S. Mahmoud, "Detection of High Impedance Faults in M.V. Mesh Distribution Network, Modern Electric Power Systems”, IEEE MEPS, 2015. DOI: 10.1109/meps.2015.7477219.

[4] Muhammad M.A.S. Mahmoud, 3-Phase Fault Finding in Oil Field MV Distribution Network Using Fuzzy Clustering Techniques, Journal of Energy and Power Engineering 2013.

[5] Muhammad M.A.S. Mahmoud "Fault Location in Multi-ring Distribution Network Using Artificial Neural Network", Journal of Electric Power Systems Research 2003. DOI: 10.1016/s0378-7796(02)00174-8.

[6] Nigel Hampton, "High and EHV cable Aging and Testing Issues", Cable Diagnostic Focused Initiative (CDFI)Phase II, National Electrical Energy Testing, Georgia Tech and Research and Applications Center (NEETRAC), February 2016.

[7] Rolf Winter, ZVEI e.V., "Failure Reasons for Insulations", ZVEI German Electrical and Electronic Manufacturers' Association Technical committee of the Electrical Winding \& Insulation Systems Division, February 2016.

[8] Juan A. Martinez-Velasco, Jacinto Martin-Arnedo, "Switching Overvoltages in Power Systems", Power System Transient, Encyclopaedia of Life Support Systems (EOLSS). 2019. https://www.eolss.net/sample-chapters/c05/E639-59-06.pdf

[9] Mustafa Kizilcay, Switching Overvoltages in a 400kV cable System", 16th PSCC, Glasgow, Scotland, July 14$18,2008$.

[10] F. Palone, M. Rebolini, S. Lauria, M. Maccioni, M. Schembari, J. P. Vassallo, "Switching transients on very long HV ac cable lines: simulations and measurements on the $230 \mathrm{kV}$ Malta-Sicily Interconnector", CIGRE 2016.

[11] Maialen Boyra, "Transient Overvoltages in Cable Systems, Part 2 - Experiments on fast transients in cable systems" Master of Science Thesis, Chalmers University of Technology, Sweden 2007.

[12] M. Ghassemi, "Accelerated insulation aging due to fast, repetitive voltages: A review identifying challenges and future research needs," in IEEE Transactions on Dielectrics and Electrical Insulation, vol. 26, no. 5, pp. 15581568, Oct. 2019, doi: 10.1109/TDEI.2019.008176.

[13] Abdulsalam S. Alghamdi , Rayan K. Desuqi, “A study of expected lifetime of XLPE insulation cables working at elevated temperatures by applying accelerated thermal ageing", Heliyon Vol.6, Issue 1, E03120, JANUARY 01, 2020. https://doi.org/10.1016/j.heliyon.2019.e03120 
[14] Ravish P.Y. Mehairjan, "Application of Statistical Life Data Analysis for Cable Joints in MV Distribution Networks" Master of Science Thesis, Delft University of Technology (TU Delft), 2010.

[15] Swati Sachan, Chengke Zhou, Geraint Bevan, Babakalli Alkali, "Prediction of Power Cable Failure Rate Based on Failure History and Operational Conditions", 9th International Conference on Insulated Power Cables, Jicable'15- Versailles 21-25 June, 2015 .

[16] Z. Tang et al., "Comparison of the Weibull and the Crow-AMSAA Model in Prediction of Early Cable Joint Failures," in IEEE Transactions on Power Delivery, vol. 30, no. 6, pp. 2410-2418, Dec. 2015, doi: 10.1109/TPWRD.2015.2404926.

[17] Eyad A. Feilat, "Lifetime Assessment of Electrical Insulation", Book Chapter, Electric Field, IntechOpen, 2018. http://dx.doi.org/10.5772/intechopen.72423

[18] Zhou, Chengke; Yi, Huajie; Dong, Xiang, "Review of recent research towards power cable life cycle management”, IET High Voltage, vol. 2, no. 3, pp. 179-187. https://doi.org/10.1049/hve.2017.0037.

[19] Jeongtae Kim, Woobin Kim, Hung-Sok Park and Ji-Won Kang, "Lifetime Assessment for Oil-Paper Insulation using Thermal and Electrical Multiple Degradation", Journal of Electrical Engineering and Technolgy.2017; 12(2): 840-845, https://doi.org/10.5370/JEET.2017.12.2.840

[20] M. Plopeanu, Petru Notingher and "Underground power cable insulation electrical lifetime estimation method”, U.P.B. Sci. Bull., Series C, Vol. 75, Iss. 1, 2013.

[21] Tomasz Lech Koltunowicz, “Accelerated Insulation Aging Due to Thermal and Electrical Stresses in Future Power Grids", Thesis to obtain the degree of doctor. Delft University of Technology, 2014.

[22] Gian Carlo Montanari, Peter Morshuis, Paolo Seri and Riddhi Ghosh, "Ageing and reliability of electrical insulation: the risk of hybrid AC/DC grids”, IET Journals, High Voltage, Vol. 5 Iss. 5, pp. 620-627, 2020.

[23] Mojtaba Rabiei, Seyyed Mohammad Hassan Hosseinil, “A Method for Measuring Transient Switching Overvoltage Case study: Electricity network of Imam Khomeini Airport City”, Signal Processing and Renewable Energy September 2019, pp. 23-31.ISSN: 2588-7327, eISSN: 2588-7335.

[24] Robert G. Olsen, "High Voltage Overhead Transmission Line Electromagnetics “, Createspace Independent Publishing Platform, 2018. ISBN: 9781720894230.

[25] J. Duncan Glover, Mulukutla S. Sarma, and Thomas J. Overbye, "Power System Analysis and Design, Fifth Edition”, Publisher, Global Engineering. 2012. ISBN-13: 978-1-111-42579-1.

[26], Matlab R2021b, MathWorks . https://www.mathworks.com/help/physmod/sps/powersys/ref/pisectionline.html 